\title{
How to Draw a Travel Time Curve of Explosion Observation
}

\author{
Yasuo SaTô \\ and \\ Shuzo AsANo \\ Earthquake Research Institute, \\ University of Tolkyo
}

\begin{abstract}
In the field of seismic prospecting there is an urgent need to minimize the man power of experienced people for drawing a travel time curve of explosion observation. Otherwise the speed of the data accumulation exceeds that of the data processing, and data sit on desks permanently. Here a simple computer oriented method is suggested, which is similar to the method of least squares, but the result is not affected by points far from the straight line which is the true travel time curve.
\end{abstract}

\section{$\$ 1$. Introduction}

When a blast of explosive is carried out, we observe as many first arrivals of $P$ wave as seismographs. If we add other later phases, many more points of arrival times are included. in the chart of arrival time vs. epicentral clistance. These points, however, not necessarily sit on a small number of straight lines, but many of them are far from any of these lines. Consequently we usually throw away such points and choose only those which are likely to be on a certain straight line. This choice, however, is clone rather arbitrarily by individual experts spending much time, and yet the objectivity is sometimes doubtful. Besides, there is another difficulty in determining the straight lines, namely there are points which may be interpreted to belong to a certain straight line, but could be interpreted to belong to another. In order to solve these difficulties and save the man power of experienced people, a computer oriented method is suggested in the following sections. A similar and more elaborate method was suggested by L. Knopoff and T.L. Teng (1965) and the present work is an attempt to prepare a practical method which is easy to apply to an actual work.

\section{§2. Principle}

Suppose there is a group of points $P_{k}\left(x_{k}, y_{k}\right)$ $(k=1,2, \ldots, n)$ and it is required to prepare a straight line determined by these points. If the ordinary method of least squares is employed, the evaluation of fitness of a straight line $\mathrm{L}$ is clone by the quantity

$$
V_{o}[L]=A_{o} \exp \left(-h_{0} \sum_{k=1}^{n} \delta_{k}^{2}\right)
$$

where $\delta_{k}$ is the deviation of the point $P_{k}$ from the straight line and $V_{0}[L]$ takes the largest value when $\sum \delta_{k^{2}}{ }^{2}$ becomes the smallest. In the present problem, however, not all the $n$ points are supposed to belong to the line $L$, but only some of them do, consequently the evaluation must be done in a different way.

Here we suggest the following definition of $V[L]$, namely

$$
V[L]=A \sum_{k=1}^{n} \exp \left(-h \ddot{o}_{k}^{2}\right) .
$$

In this expression if $\delta_{k}$ is fairly large $\exp \left(-h \delta_{k}{ }^{2}\right)$ becomes very small and has little effect to $V[L]$, therefore the evaluation of fitness of $L$ is actually done by the points which are not far from the line $L$. If the points, which satisfy the condition

$$
\exp \left(-h \delta_{k}^{2}\right)<\varepsilon<1
$$

and have no actual contribution to $V[L]$, are abandoned, the expression $(2 \cdot 2)$ is modified as follows:

$$
V[L]=A \sum_{j=1}^{m} \exp \left(-h \delta_{j}^{2}\right) .
$$

$P_{j}\left(x_{j}, y_{j}\right)(j=1,2, \ldots, m)$ are the points which actually take part in the determination of $L$. 
In order to secure the validity of this choosing results. Adjusting the parameters of the process, $(2 \cdot 4)$ from $(2 \cdot 2)$, we may try several straight line we can find the line $L$ that makes combinations of $h$ and $\varepsilon$, and compare the the value $V[L]$ maximum.

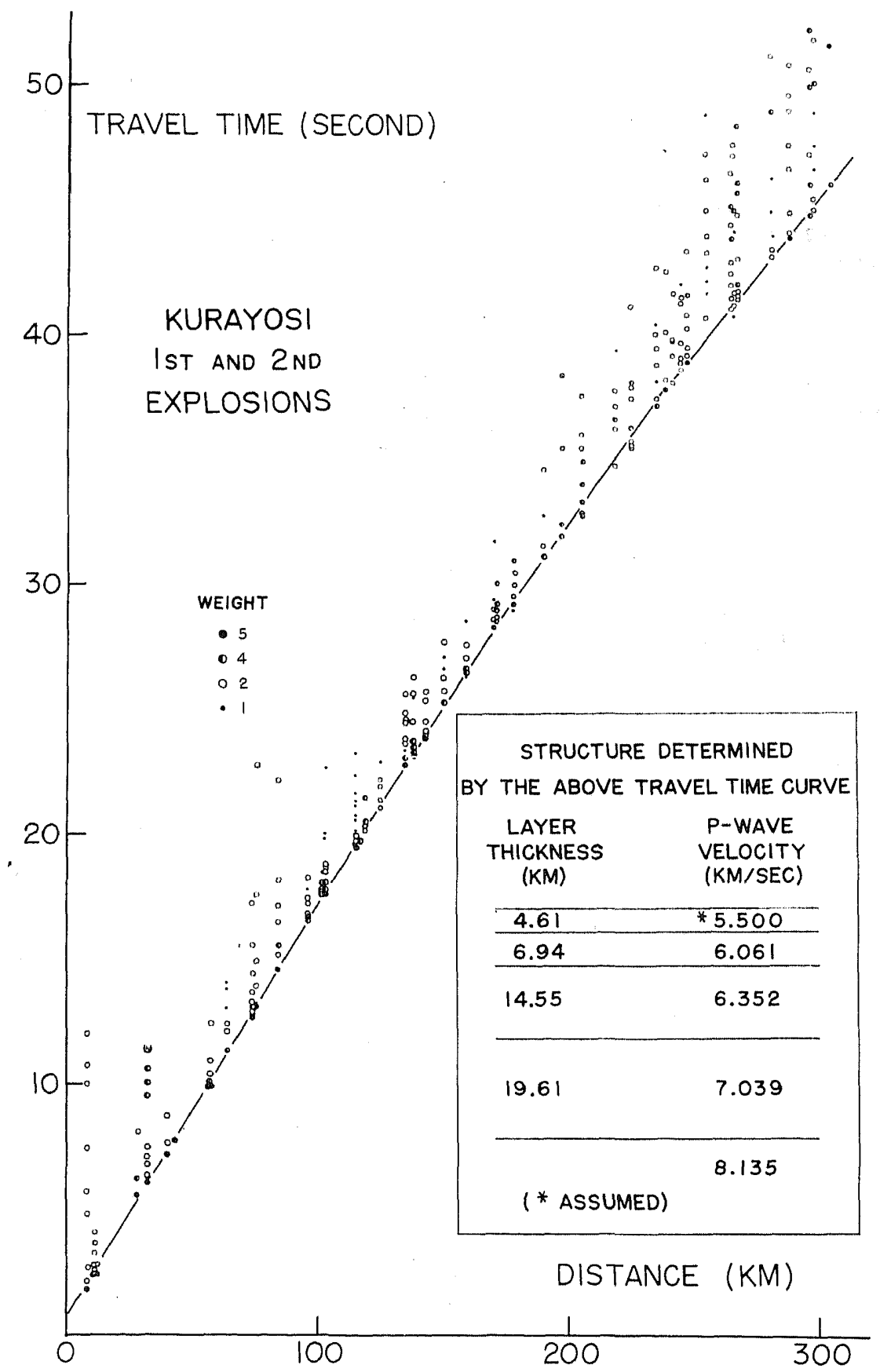

Fig. 1. Travel time curves and structure obtained by the present method. 
In order to take the goodness or the clearness of the onsets of phases into account, we can give a weight $w_{k}$ to each reading. In that case the value $V[L]$ is calculated as follows instead of $(2 \cdot 2)$ :

$$
V[L]=A \sum_{k} w_{k} \exp \left(-h \delta_{k}^{2}\right)
$$

Here we may add that as long as the argument is small and exp-function can be approximated by

$$
\exp \left(-h \delta^{2}\right)=C-q \delta^{2}
$$

$(2 \cdot 2)$ or $(2 \cdot 5)$ proves to be nothing but the method of least squares itself.

Although the discussions have been confined so far to the fitting of a straight line to the observed data, it will be worth to note that the above argument can be applied to the fitting of any curve given by a function $f(\Delta)$ of the epicentral distance $\Delta$. In such a case we only substitute $\delta_{k}$ by ( $k$-th observed value $\left.-f\left(\Delta_{k}\right)\right)$.

\section{§3. Actual procedure}

In the actual procedure we still have some difficulty, because we have to choose a number of plausible straight lines, which are to be tested. A computer oriented way of solving this difficulty is to try at first all the straight lines determined by the possible combinations of two points $\mathrm{P}_{r}$ and $\mathrm{P}_{s}(r, s=1,2, \cdots, n$; $r \neq s)$ and then pick out only those lines which have high evaluations $V[L]$. After that we can recalculate the precise equation for the lines $L$ as indicated in the previous section.

In Fig. 1 an example is given, the data of which are the arrival times of the first and second Kurayosi explosions (1966). The readings of phases are classified into four grades
$A, B, C$ and $D$ according to the accuracy of identification and clearness of phases. The scatter of observed travel times is about \pm 0.1 $\mathrm{sec}$ and less than $\pm 0.2 \mathrm{sec}$. Assigning the weight $1.0,0.8,0.4$ and 0.2 for the grades $A$, $\mathrm{B}, \mathrm{C}$ and $\mathrm{D}$ respectively, the following solution was obtained and is shown in Fig. 1.

$\begin{array}{cccc}\begin{array}{c}\text { Apparent } \\ \text { Velocity } \\ (\mathrm{km} / \mathrm{sec})\end{array} & \begin{array}{c}\text { Intercept } \\ \text { Time } \\ (\mathrm{sec})\end{array} & \begin{array}{c}\text { Data } \\ \text { (Distance) } \\ (\mathrm{km})\end{array} & \begin{array}{c}\text { Number of } \\ \text { Data } \\ \text { Points }\end{array} \\ 6.060 & 0.704 & 1-120 & 108 \\ 6.352 & 1.523 & 110-160 & 68 \\ 7.039 & 4.184 & 170-250 & 77 \\ 8.135 & 8.416 & 230-305 & 98\end{array}$

The data are divided into four groups as given above, but this is only to minimize the computing time and is not a necessary procedure. For example, if all the data are used at a time and the velocity around $6 \mathrm{~km} / \mathrm{sec}$ is searched for, the answer comes out as follows and there is no essential difference from the value given in the first line above:

Apparent velocity $=6.065 \mathrm{~km} / \mathrm{sec}$,

Intercept time $=0.712 \mathrm{sec}$.

One of the solutions given by Hashizume et al (1966) is close to the above solution. The difference may be due to the fact that they neglected near-by observation and also they took data of the reverse profile into consideration.

\section{References}

Hashizume, M., O. Kawamoto, S. Asano, I. Muramatu, T. Asada, I. Tamakr and S. Murauchr: Bull. Earthq. Res. Inst., 44 (1966) 109.

Knoporf, L. and T. L. TenG:

Rev. Geophys., 3 (1965), 11-24.

The Research Group for Explosion Seismology, Bull, Earthq. Res. Inst., 44 (1966) 89. 